\title{
A Note on "A Lexicographic Method for Matrix Games with Payoffs of Triangular Intuitionistic Fuzzy Numbers"
}

\author{
Tina Verma* \\ School of Mathematics and Computer Applications, Thapar University, \\ Patiala 147004, Punjab, India \\ E-mail:verma.tina21@gmail.com \\ Amit Kumar \\ School of Mathematics and Computer Applications, Thapar University, \\ Patiala 147004, Punjab, India \\ E-mail: amitkdma@gmail.com \\ www.thapar.edu
}

Received 11 November 2013

Accepted 10 March 2015

\begin{abstract}
Nan et al. [J.-X. Nan, D.-F. Li and M.-J. Zhang, A lexicographic method for matrix games with payoffs of triangular intuitionistic fuzzy numbers, International Journal of Computational Intelligence Systems 3(3) (2010) 280-289] pointed out that there is no method in the literature to find the solution of such matrix games in which payoffs are represented by triangular intuitionistic fuzzy numbers and proposed a method for the same. In this paper, it is pointed out that Nan et al. have used some mathematical incorrect assumptions in their proposed method and the existing method is also modified.
\end{abstract}

Keywords: Triangular intuitionistic fuzzy number; Intuitionistic fuzzy set; Matrix game; Mathematical programming; Lexicographic method.

\section{Existing Method for Comparing Triangular Intuitionistic Fuzzy Numbers}

In this section, the method, used by Nan et al. [1] for comparing triangular intuitionistic fuzzy numbers, is presented [1, Section 2.2, Definition 7, pp. 283].

If $\tilde{a}_{1}=\left\langle\left(\underline{a}_{1}, a_{1}, \bar{a}_{1}\right) ; w_{\tilde{u}_{1}}, u_{\tilde{a}_{1}}\right\rangle$ and $\tilde{a}_{2}=\left\langle\left(\underline{a}_{2}, a_{2}, \bar{a}_{2}\right) ; w_{\tilde{a}_{2}}, u_{\tilde{a}_{2}}\right\rangle$ are two triangular intuitionistic fuzzy numbers. Then,

$$
\text { i. } \quad \tilde{a}_{1}>_{I F} \tilde{a}_{2} \quad \text { if } \quad S_{\mu}\left(\tilde{a}_{1}\right)>S_{\mu}\left(\tilde{a}_{2}\right) \text { or } \quad \text { if }
$$

$S_{\mu}\left(\tilde{a}_{1}\right)=S_{\mu}\left(\tilde{a}_{2}\right)$ then $S_{v}\left(\tilde{a}_{1}\right)>S_{v}\left(\tilde{a}_{2}\right)$.

ii. $\quad \tilde{a}_{1}=_{I F} \tilde{a}_{2}$ if and only if $S_{\mu}\left(\tilde{a}_{1}\right)=S_{\mu}\left(\tilde{a}_{2}\right)$ and $S_{v}\left(\tilde{a}_{1}\right)=S_{v}\left(\tilde{a}_{2}\right)$. where, $S_{\mu}\left(\tilde{a}_{i}\right)=\frac{\left(w_{\tilde{a}_{i}}\right)\left(2 a_{i}+\underline{a}_{i}+\bar{a}_{i}\right)}{4}$ and $S_{v}\left(\tilde{a}_{i}\right)=\frac{\left(1-u_{\tilde{a}_{i}}\right)\left(2 a_{i}+\underline{a}_{i}+\bar{a}_{i}\right)}{4}$, are called membership and non-membership function average indexes respectively.

\section{Existing Method}

Nan et al. [1] proposed the following method to find the optimal solution (maximin strategy for player 1, minimax strategy for player 2 and value of intuitionistic fuzzy matrix game for player 1) of such intuitionistic

* Corresponding address: School of Mathematics and Computer Applications, Thapar University, Nabha Road, Patiala 147004, Punjab, India. 
fuzzy matrix games in which payoffs are represented by triangular intuitionistic fuzzy numbers.

Step 1: Formulate the chosen problem as the mathematical programming problems $\mathrm{P} 1$ and $\mathrm{P} 2$ to find the maximin strategy $y_{i}^{*}, i=1,2, \ldots m$ and minimax strategy $z_{j}^{*}, j=1,2, \ldots n$ for player 1 and player 2 respectively.

\section{Problem P1 [1, Equation 3, pp. 284]}

$\operatorname{Maximize}(\tilde{v})$

Subject to

$\sum_{i=1}^{m} \tilde{a}_{i j} y_{i} \geq_{I F} \tilde{v}, j=1,2, \ldots, n$;

$\sum_{i=1}^{m} y_{i}=1$

$y_{i} \geq 0, i=1,2, \ldots, m$.

Problem P2 [1, Equation 4, pp. 284]

$\operatorname{Minimize}(\tilde{\omega})$

Subject to

$\sum_{j=1}^{n} \tilde{a}_{i j} z_{j} \leq_{I F} \tilde{\omega}, i=1,2, \ldots, m ;$

$\sum_{j=1}^{n} z_{j}=1$;

$z_{j} \geq 0, j=1,2, \ldots, n$.

Step 2: Using Section 1, the problems P1 and P2 can be transformed into problems $\mathrm{P} 3$ and $\mathrm{P} 4$ respectively.

Problem P3 [1, Equation 5, pp. 284]

$\operatorname{Maximize}\left\{S_{\mu}(\tilde{v}), S_{v}(\tilde{v})\right\}$

Subject to

$S_{\mu}\left(\sum_{i=1}^{m} \tilde{a}_{i j} y_{i}\right) \geq S_{\mu}(\tilde{v}), \quad j=1,2, \ldots, n ;$

$S_{v}\left(\sum_{i=1}^{m} \tilde{a}_{i j} y_{i}\right) \geq S_{v}(\tilde{v}), \quad j=1,2, \ldots, n ;$

$\sum_{i=1}^{m} y_{i}=1$;

$y_{i} \geq 0, i=1,2, \ldots, m$.

Problem P4 [1, Equation 10, pp. 286]

$\operatorname{Minimize}\left\{S_{\mu}(\tilde{\omega}), S_{v}(\tilde{\omega})\right\}$

Subject to

$S_{\mu}\left(\sum_{j=1}^{n} \tilde{a}_{i j} z_{j}\right) \leq S_{\mu}(\tilde{\omega}), i=1,2, \ldots, m ;$
$S_{\nu}\left(\sum_{j=1}^{n} \tilde{a}_{i j} z_{j}\right) \leq S_{\nu}(\tilde{\omega}), i=1,2, \ldots, m$

$\sum_{j=1}^{n} z_{j}=1$

$z_{j} \geq 0, j=1,2, \ldots, n$.

Step 3: Using the properties $S_{\mu}\left(\sum_{i=1}^{n} \tilde{a}_{i}\right)=\sum_{i=1}^{n} S_{\mu}\left(\tilde{a}_{i}\right)$ and $S_{v}\left(\sum_{i=1}^{n} \tilde{a}_{i}\right)=\sum_{i=1}^{n} S_{v}\left(\tilde{a}_{i}\right)$, the problems $\mathrm{P} 3$ and $\mathrm{P} 4$ can be transformed into problems P5 and P6 respectively.

Problem P5 [1, Equation 6, pp. 285]

Maximize $\left\{S_{\mu}(\tilde{v}), S_{v}(\tilde{v})\right\}$

Subject to

$\sum_{i=1}^{m} S_{\mu}\left(\tilde{a}_{i j} y_{i}\right) \geq S_{\mu}(\tilde{v}), j=1,2, \ldots, n ;$

$\sum_{i=1}^{m} S_{v}\left(\tilde{a}_{i j} y_{i}\right) \geq S_{v}(\tilde{v}), j=1,2, \ldots, n ;$

$\sum_{i=1}^{m} y_{i}=1$

$y_{i} \geq 0, i=1,2, \ldots, m$.

Problem P6 [1, Equation 11, pp. 286]

$\operatorname{Minimize}\left\{S_{\mu}(\tilde{\omega}), S_{v}(\tilde{\omega})\right\}$

Subject to

$\sum_{j=1}^{n} S_{\mu}\left(\tilde{a}_{i j} z_{j}\right) \leq S_{\mu}(\tilde{\omega}), i=1,2, \ldots, m ;$

$\sum_{j=1}^{n} S_{v}\left(\tilde{a}_{i j} z_{j}\right) \leq S_{v}(\tilde{\omega}), i=1,2, \ldots, m ;$

$\sum_{j=1}^{n} z_{j}=1$

$z_{j} \geq 0, j=1,2, \ldots, n$.

Step 4: Putting $S_{\mu}(\tilde{a})=\frac{\left(w_{\tilde{a}}\right)(2 a+\underline{a}+\bar{a})}{4} \quad$ and $S_{v}(\tilde{a})=\frac{\left(1-u_{\tilde{a}}\right)(2 a+\underline{a}+\bar{a})}{4}$, the problems P5 and P6 can be transformed into problems P7 and P8 respectively.

Problem P7 [1, Equation 6, pp. 285]

$\operatorname{Maximize}\left\{\left(w_{\tilde{v}}\right) \frac{(\underline{v}+2 v+\bar{v})}{4},\left(1-u_{\tilde{v}}\right) \frac{(\underline{v}+2 v+\bar{v})}{4}\right\}$

Subject to 
$\sum_{i=1}^{m} \frac{\left(w_{\tilde{a}_{i j}}\right)\left(\underline{a}_{i j}+2 a_{i j}+\bar{a}_{i j}\right) y_{i}}{4} \geq\left(w_{\tilde{v}}\right) \frac{(\underline{v}+2 v+\bar{v})}{4}, j=1,2, \ldots n ;$

$\sum_{i=1}^{m} \frac{\left(1-u_{\tilde{a}_{i j}}\right)\left(\underline{a}_{i j}+2 a_{i j}+\bar{a}_{i j}\right) y_{i}}{4} \geq$

$$
\left(1-u_{\tilde{v}}\right) \frac{(\underline{v}+2 v+\bar{v})}{4}, j=1,2, \ldots n
$$

$\underline{v} \leq v$

$v \leq \bar{v}$;

$\sum_{i=1}^{m} y_{i}=1$;

$y_{i} \geq 0, \quad i=1,2, \ldots, m$.

where, $w_{\tilde{v}}=\min _{\substack{1 \leq i \leq m \\ 1 \leq j \leq n}}\left\{w_{\tilde{a}_{u_{j}}}\right\}$ and $u_{\tilde{v}}=\max _{\substack{1 \leq i \leq m \\ 1 \leq j \leq n}}\left\{u_{\tilde{a}_{i_{j}}}\right\}$.

Problem P8 [1, Equation 11, pp. 286]

Minimize $\left\{\left(w_{\tilde{\omega}}\right) \frac{(\underline{\omega}+2 \omega+\bar{\omega})}{4},\left(1-u_{\tilde{\omega}}\right) \frac{(\underline{\omega}+2 \omega+\bar{\omega})}{4}\right\}$

Subject to

$\sum_{j=1}^{n} \frac{\left(w_{\tilde{a}_{i j}}\right)\left(\underline{a}_{i j}+2 a_{i j}+\bar{a}_{i j}\right) z_{j}}{4} \leq\left(w_{\tilde{\omega}}\right) \frac{(\underline{\omega}+2 \omega+\bar{\omega})}{4}, i=1,2, \ldots m ;$

$\sum_{j=1}^{n} \frac{\left(1-u_{\tilde{a}_{j}}\right)\left(\underline{a}_{i j}+2 a_{i j}+\bar{a}_{i j}\right) z_{j}}{4} \leq$

$$
\left(1-u_{\tilde{\omega}}\right) \frac{(\underline{\omega}+2 \omega+\bar{\omega})}{4}, i=1,2, \ldots m ;
$$

$\underline{\omega} \leq \omega$

$\omega \leq \bar{\omega}$;

$\sum_{j=1}^{n} z_{j}=1$;

$z_{j} \geq 0, \quad j=1,2, \ldots, n$.

where, $w_{\tilde{\omega}}=\min _{\substack{1 \leq i \leq m \\ 1 \leq j \leq n}}\left\{w_{\tilde{a}_{i_{j}}}\right\}$ and $u_{\tilde{\omega}}=\max _{\substack{1 \leq i \leq m \\ 1 \leq j \leq n}}\left\{u_{\tilde{a}_{\tilde{i}}}\right\}$.

Step 5: Assuming

$\left(w_{\tilde{v}}\right) \frac{(\underline{v}+2 v+\bar{v})}{4}=v_{1},\left(1-u_{\tilde{v}}\right) \frac{(\underline{v}+2 v+\bar{v})}{4}=v_{2}$ and

$\left(w_{\tilde{\omega}}\right) \frac{(\underline{\omega}+2 \omega+\bar{\omega})}{4}=\omega_{1},\left(1-u_{\tilde{\omega}}\right) \frac{(\underline{\omega}+2 \omega+\bar{\omega})}{4}=\omega_{2}$, the

problems P7 and P8 can be transformed into problems $\mathrm{P} 9$ and $\mathrm{P} 10$ respectively.

Problem P9 [1, Equation 7, pp. 285]

Maximize $\left\{v_{1}, v_{2}\right\}$

Subject to $\sum_{i=1}^{m}\left(\frac{\left(w_{\tilde{a}_{i j}}\right)\left(\underline{a}_{i j}+2 a_{i j}+\bar{a}_{i j}\right) y_{i}}{4}\right) \geq v_{1}, j=1,2, \ldots n ;$

$\sum_{i=1}^{m}\left(\frac{\left(1-u_{\tilde{a}_{j}}\right)\left(\underline{a}_{i j}+2 a_{i j}+\bar{a}_{i j}\right) y_{i}}{4}\right) \geq v_{2}, j=1,2, \ldots n ;$

$v_{2} \geq v_{1}$

$\sum_{i=1}^{m} y_{i}=1$

$y_{i} \geq 0, i=1,2, \ldots, m$.

\section{Problem P10 [1, Equation 12, pp. 286]}

Minimize $\left\{\omega_{1}, \omega_{2}\right\}$

Subject to

$\sum_{j=1}^{n}\left(\frac{\left(w_{\tilde{a}_{j}}\right)\left(\underline{a}_{i j}+2 a_{i j}+\bar{a}_{i j}\right) z_{j}}{4}\right) \leq \omega_{1}, i=1,2, \ldots m ;$

$\sum_{j=1}^{n}\left(\frac{\left(1-u_{\tilde{a}_{j}}\right)\left(\underline{a}_{i j}+2 a_{i j}+\bar{a}_{i j}\right) z_{j}}{4}\right) \leq \omega_{2}, i=1,2, \ldots m ;$

$\omega_{1} \leq \omega_{2} ;$

$\sum_{j=1}^{n} z_{j}=1$

$z_{j} \geq 0, \quad j=1,2, \ldots, n$.

Step 6: Using lexicographic method the optimal solution of problems P9 and P10 and hence the optimal solution of problems $\mathrm{P} 1$ and $\mathrm{P} 2$ respectively can be obtained as follows.

Step 6(a): Find the optimal solution $\left\{v_{1}, v_{2}, y_{i}, i=1,2, \ldots, m\right\}$ and $\left\{\omega_{1}, \omega_{2}, z_{j}, j=1,2, \ldots, n\right\}$ of problems $\mathrm{P} 11$ and $\mathrm{P} 12$ respectively. Let it be denoted by $\left\{v_{1}^{0}, v_{2}^{0}, y_{i}^{0}, i=1,2, \ldots, m\right\}$ and $\quad\left\{\omega_{1}^{0}, \omega_{2}^{0}, z_{j}^{0}, j=1,2, \ldots, n\right\}$ respectively.

Problem P11 [1, Equation 8, pp. 285]

Maximize $\left\{v_{1}\right\}$

Subject to

Constraints of problem P9.

Problem P12 [1, Equation 13, pp. 286]

Minimize $\left\{\omega_{1}\right\}$

Subject to

Constraints of problem P10.

Step 6(b): Find the optimal solution 
$\left\{v_{1}, v_{2}, y_{i}, i=1,2, \ldots, m\right\}$ and $\left\{\omega_{1}, \omega_{2}, z_{j}, j=1,2, \ldots, n\right\}$ of problems $\mathrm{P} 13$ and $\mathrm{P} 14$ respectively. Let it be denoted by $\left\{v_{1}^{*}, v_{2}^{*}, y_{i}^{*}, i=1,2, \ldots, m\right\}$ and $\left\{\omega_{1}^{*}, \omega_{2}^{*}, z_{j}^{*}, j=1,2, \ldots, n\right\}$ respectively.

\section{Problem P13 [1, Equation 9, pp. 285]}

Maximize $\left\{v_{2}\right\}$

Subject to

Constraints of problem P9 with additional constraints $v_{1} \geq v_{1}^{0}$;

$v_{2} \geq v_{2}^{0}$.

\section{Problem P14 [1, Equation 14, pp. 286]}

Minimize $\left\{\omega_{2}\right\}$

Subject to

Constraints of problem P10 with additional constraints $\omega_{1} \leq \omega_{1}^{0}$;

$\omega_{2} \leq \omega_{2}^{0}$.

Step 7: Using the maximin strategy $y_{i}^{*}, i=1,2, \ldots m$ and minimax strategy $z_{j}^{*}, j=1,2, \ldots n$, obtained in Step 6(b), the value of intuitionistic fuzzy matrix game for player1 is $E\left(y_{i}^{*}, z_{j}^{*}\right)=\sum_{i=1}^{m} \sum_{j=1}^{n} y_{i}^{*} \tilde{a}_{i j} z_{j}^{*}$.

\section{Error in the Existing Method}

If $\tilde{a}_{i}=\left\langle\left(\underline{a}_{i}, a_{i}, \bar{a}_{i}\right) ; w_{\tilde{a}_{i}}, u_{\tilde{a}_{i}}\right\rangle, i=1,2, \ldots, n$ are $n$ triangular intuitionistic fuzzy numbers then using Definition 2 [1, Section 2.1, pp. 282],

(i) $\sum_{i=1}^{n} \tilde{a}_{i}=\sum_{i=1}^{n}\left(\left\langle\left(\underline{a}_{i}, a_{i}, \bar{a}_{i}\right) ; w_{\tilde{a}_{i}}, u_{\tilde{a}_{i}}\right\rangle\right)$

$$
=\left\langle\left(\sum_{i=1}^{n} \underline{a}_{i}, \sum_{i=1}^{n} a_{i}, \sum_{i=1}^{n} \bar{a}_{i}\right) ; \min _{1 \leq i \leq n}\left(w_{\tilde{a}_{i}}\right), \max _{1 \leq i \leq n}\left(u_{\tilde{a}_{i}}\right)\right\rangle .
$$

(ii) $\lambda \tilde{a}_{i}=\lambda\left\langle\left(\underline{a}_{i}, a_{i}, \bar{a}_{i}\right) ; w_{a_{i}}, u_{a_{i}}\right\rangle=$

$$
= \begin{cases}\left\langle\left(\lambda \underline{a}_{i}, \lambda a_{i}, \lambda \bar{a}_{i}\right) ; w_{\tilde{a}_{i}}, u_{\tilde{a}_{i}}\right\rangle, & \lambda \geq 0, \\ \left\langle\left(\lambda \bar{a}_{i}, \lambda a_{i}, \lambda \underline{a}_{i}\right) ; w_{\tilde{a}_{i}}, u_{\tilde{a}_{i}}\right\rangle, & \lambda<0 .\end{cases}
$$

Therefore,

$$
\begin{aligned}
& S_{\mu}\left(\sum_{i=1}^{n} \tilde{a}_{i}\right)=\min _{1 \leq i \leq n}\left(w_{\tilde{a}_{i}}\right) \frac{\left(\sum_{i=1}^{n} \underline{a}_{i}+2 \sum_{i=1}^{n} a_{i}+\sum_{i=1}^{n} \bar{a}_{i}\right)}{4}, \\
& S_{\nu}\left(\sum_{i=1}^{n} \tilde{a}_{i}\right)=\left(1-\max _{1 \leq i \leq n}\left(u_{\tilde{a}_{i}}\right)\right) \frac{\left(\sum_{i=1}^{n} \underline{a}_{i}+2 \sum_{i=1}^{n} a_{i}+\sum_{i=1}^{n} \bar{a}_{i}\right)}{4}
\end{aligned}
$$

and $\sum_{i=1}^{n} S_{\mu}\left(\tilde{a}_{i}\right)=\sum_{i=1}^{n} \frac{\left(w_{\tilde{a}_{i}}\right)\left(\underline{a}_{i}+2 a_{i}+\bar{a}_{i}\right)}{4}$,

$\sum_{i=1}^{n} S_{\nu}\left(\tilde{a}_{i}\right)=\sum_{i=1}^{n} \frac{\left(1-u_{\tilde{a}_{i}}\right)\left(\underline{a}_{i}+2 a_{i}+\bar{a}_{i}\right)}{4}$.

It is obvious that $S_{\mu}\left(\sum_{i=1}^{n} \tilde{a}_{i}\right) \neq \sum_{i=1}^{n} S_{\mu}\left(\tilde{a}_{i}\right)$

and $S_{v}\left(\sum_{i=1}^{n} \tilde{a}_{i}\right) \neq \sum_{i=1}^{n} S_{v}\left(\tilde{a}_{i}\right)$

However, in Step 3 of the existing method, described in Section 2, for transforming problems P3 and P4 into problems P5 and P6 respectively, Nan et al. [1] have used the mathematical properties $S_{\mu}\left(\sum_{i=1}^{m} \tilde{a}_{i j} y_{i}\right)=\sum_{i=1}^{m} S_{\mu}\left(\tilde{a}_{i j} y_{i}\right), S_{v}\left(\sum_{i=1}^{m} \tilde{a}_{i j} y_{i}\right)=\sum_{i=1}^{m} S_{v}\left(\tilde{a}_{i j} y_{i}\right)$ and

$S_{\mu}\left(\sum_{j=1}^{n} \tilde{a}_{i j} z_{j}\right)=\sum_{j=1}^{n} S_{\mu}\left(\tilde{a}_{i j} z_{j}\right), S_{\nu}\left(\sum_{j=1}^{n} \tilde{a}_{i j} z_{j}\right)=\sum_{j=1}^{n} S_{\nu}\left(\tilde{a}_{i j} z_{j}\right)$ respectively, which do not hold in general. Hence, there is error in the existing method [1].

\section{Modified Method}

In this section, the existing method [1] is modified and the steps are as follows.

Step 1: Assuming $\tilde{a}_{i j}=\left\langle\left(\underline{a}_{i j}, a_{i j}, \bar{a}_{i j}\right) ; w_{\tilde{a}_{i j}}, u_{\tilde{a}_{i j}}\right\rangle$, $\tilde{v}=\left\langle(\underline{v}, v, \bar{v}) ; w_{\tilde{v}}, u_{\tilde{v}}\right\rangle$ and $\tilde{\omega}=\left\langle(\underline{\omega}, \omega, \bar{\omega}) ; w_{\tilde{\omega}}, u_{\tilde{\omega}}\right\rangle$, where $w_{\tilde{v}}=w_{\tilde{\omega}}=\min _{\substack{1 \leq i \leq m \\ 1 \leq j \leq n}}\left\{w_{\tilde{a}_{i}}\right\}$ and $u_{\tilde{v}}=u_{\tilde{\omega}}=\max _{\substack{1 \leq i \leq m \\ 1 \leq j \leq n}}\left\{u_{\tilde{a}_{i j}}\right\}$, the problems $\mathrm{P} 1$ and $\mathrm{P} 2$ can be transformed into problems $\mathrm{P} 15$ and $\mathrm{P} 16$ respectively.

\section{Problem P15}

$\operatorname{Maximize}\left(\left\langle(\underline{v}, v, \bar{v}) ; w_{\tilde{v}}, u_{\tilde{v}}\right\rangle\right)$

Subject to

$\sum_{i=1}^{m}\left\langle\left(\underline{a}_{i j}, a_{i j}, \bar{a}_{i j}\right) ; w_{\tilde{a}_{j}}, u_{\tilde{a}_{i j}}\right\rangle y_{i} \geq_{I F}\left\langle(\underline{v}, v, \bar{v}) ; w_{\tilde{v}}, u_{\tilde{v}}\right\rangle, j=1,2, \ldots, n ;$

$\sum_{i=1}^{m} y_{i}=1$

$y_{i} \geq 0, i=1,2, \ldots, m$.

\section{Problem P16}

$\operatorname{Minimize}\left(\left\langle(\underline{\omega}, \omega, \bar{\omega}) ; w_{\tilde{\omega}}, u_{\tilde{\omega}}\right\rangle\right)$

Subject to 
$\sum_{j=1}^{n}\left\langle\left(a_{i j}, a_{i j}, \bar{a}_{i j}\right) ; w_{\tilde{a}_{j}}, u_{\tilde{u}_{i}}\right) z_{j} \leq_{I F}\left\langle(\underline{\omega}, \omega, \bar{\omega}) ; w_{\tilde{\omega}}, u_{\tilde{\omega}}\right\rangle, i=1,2, \ldots, m ;\left\langle\left(\sum_{i=1}^{m} \underline{a}_{i j} y_{i}, \sum_{i=1}^{m} a_{i j} y_{i}, \sum_{i=1}^{m} \bar{a}_{i j} y_{i}\right) ; \min _{1 \leq i \leq m}\left(w_{\tilde{a}_{j}}\right), \max _{1 \leq i \leq m}\left(u_{\tilde{a}_{j}}\right)\right\rangle \geq_{I F}$

$\sum_{j=1}^{n} z_{j}=1 ;$

$\left\langle(\underline{v}, v, \bar{v}) ; w_{\bar{v}}, u_{\tilde{v}}\right\rangle, j=1,2, \ldots, n ;$

$z_{j} \geq 0, j=1,2, \ldots, n$.

$\sum_{i=1}^{m} y_{i}=1$

Step 2: Using the arithmetic operation $\left\langle(\underline{a}, a, \bar{a}) ; w_{\tilde{a}}, u_{\tilde{a}}\right\rangle \lambda=\left\langle(\underline{a} \lambda, a \lambda, \bar{a} \lambda) ; w_{\tilde{a}}, u_{\tilde{a}}\right\rangle, \lambda \geq 0$, the $y_{i} \geq 0, i=1,2, \ldots, m$.

problems $\mathrm{P} 15$ and P16 can be transformed into problems P17 and P18 respectively.

\section{Problem P17}

$\operatorname{Maximize}\left(\left\langle(\underline{v}, v, \bar{v}) ; w_{\bar{v}}, u_{\vec{v}}\right\rangle\right)$

Subject to

$\sum_{i=1}^{m}\left\langle\left(a_{i j} y_{i}, a_{i j} y_{i}, \bar{a}_{i j} y_{i}\right) ; w_{\tilde{u}_{j}}, u_{\tilde{a}_{j}}\right\rangle \geq_{I F}$

$$
\left\langle(\underline{v}, v, \bar{v}) ; w_{\bar{v}}, u_{\tilde{v}}\right\rangle, j=1,2, \ldots, n ;
$$

$\sum_{i=1}^{m} y_{i}=1 ;$

$y_{i} \geq 0, i=1,2, \ldots, m$.

\section{Problem P18}

$\operatorname{Minimize}\left(\left\langle(\underline{\omega}, \omega, \bar{\omega}) ; w_{\tilde{\omega}}, u_{\tilde{\omega}}\right\rangle\right)$

Subject to

$\sum_{j=1}^{n}\left\langle\left(a_{i j} z_{j}, a_{i j} z_{j}, \bar{a}_{i j} z_{j}\right) ; w_{\tilde{a}_{i}}, u_{\tilde{a}_{i j}}\right\rangle \leq_{I F}$

$\left\langle(\underline{\omega}, \omega, \bar{\omega}) ; w_{\tilde{\omega}}, u_{\tilde{\omega}}\right\rangle, i=1,2, \ldots, m ;$

$\sum_{j=1}^{n} z_{j}=1$;

$z_{j} \geq 0, j=1,2, \ldots, n$.

Step 3: Using the arithmetic operation

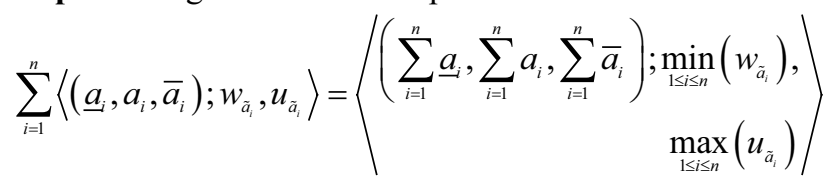

, the problems P17 and P18 can be transformed into problems P19 and P20 respectively.

\section{Problem P19}

$\operatorname{Maximize}\left(\left\langle(\underline{v}, v, \bar{v}) ; w_{\bar{v}}, u_{\bar{v}}\right\rangle\right)$

Subject to

\section{Problem P20}

Minimize $\left\langle(\underline{\omega}, \omega, \bar{\omega}) ; w_{\tilde{\omega}}, u_{\tilde{\omega}}\right\rangle$

Subject to

$$
\begin{aligned}
& \begin{array}{l}
\left\langle\left(\sum_{j=1}^{n} \underline{a}_{j j} z_{j}, \sum_{j=1}^{n} a_{i j} z_{j}, \sum_{j=1}^{n} \bar{a}_{i j} z_{j}\right) ; \min _{1 \leq j \leq n}\left(w_{\tilde{a}_{u}}\right), \max _{1 \leq j \leq n}\left(u_{\tilde{a}_{j}}\right)\right\rangle \leq_{I F} \\
\left\langle(\underline{\omega}, \omega, \bar{\omega}) ; w_{\tilde{\omega}}, u_{\tilde{\omega}}\right\rangle, i=1,2, \ldots, m ;
\end{array} \\
& \sum_{j=1}^{n} z_{j}=1 ; \\
& z_{j} \geq 0, j=1,2, \ldots, n .
\end{aligned}
$$

Step 4: Using Section 1, the problems P19 and P20 can be transformed into problems P21 and P22 respectively.

\section{Problem P21}

\section{Maximize}

$\left\{S_{\mu}\left(\left\langle(\underline{v}, v, \bar{v}) ; w_{\bar{v}}, u_{\tilde{v}}\right\rangle\right), S_{\nu}\left(\left\langle(\underline{v}, v, \bar{v}) ; w_{\tilde{v}}, u_{\tilde{v}}\right\rangle\right)\right\}$

Subject to

$$
\begin{array}{r}
S_{\mu}\left(\left\langle\left(\sum_{i=1}^{m} \underline{a}_{i j} y_{i}, \sum_{i=1}^{m} a_{i j} y_{i}, \sum_{i=1}^{m} \bar{a}_{i j} y_{i}\right) ; \min _{1 \leq i \leq m}\left(w_{\bar{a}_{v}}\right), \max _{1 \leq i \leq m}\left(u_{\bar{a}_{i}}\right)\right\rangle\right) \geq \\
S_{\mu}\left(\left\langle(\underline{v}, v, \bar{v}) ; w_{\bar{v}}, u_{\bar{v}}\right\rangle\right), j=1,2, \ldots, n ; \\
S_{v}\left(\left\langle\left(\sum_{i=1}^{m} a_{i j} y_{i}, \sum_{i=1}^{m} a_{i j} y_{i}, \sum_{i=1}^{m} \bar{a}_{i j} y_{i}\right) ; \min _{1 \leq i \leq m}\left(w_{\bar{u}_{v}}\right), \max _{1 \leq i \leq m}\left(u_{\bar{a}_{i}}\right)\right\rangle\right) \geq \\
S_{v}\left(\left\langle(\underline{v}, v, \bar{v}) ; w_{\bar{v}}, u_{\bar{v}}\right\rangle\right), j=1,2, \ldots, n ;
\end{array}
$$

$\sum_{i=1}^{m} y_{i}=1$

$y_{i} \geq 0, i=1,2, \ldots, m$.

\section{Problem P22}

Minimize

$\left\{S_{\mu}\left(\left\langle(\underline{\omega}, \omega, \bar{\omega}) ; w_{\tilde{\omega}}, u_{\tilde{\omega}}\right\rangle\right), S_{v}\left(\left\langle(\underline{\omega}, \omega, \bar{\omega}) ; w_{\tilde{\omega}}, u_{\tilde{\omega}}\right)\right)\right\}$

Subject to

$S_{\mu}\left(\left\langle\left(\sum_{j=1}^{n} \underline{a}_{i j} z_{j}, \sum_{j=1}^{n} a_{i j} z_{j}, \sum_{j=1}^{n} \bar{a}_{i j} z_{j}\right) ; \min _{1 \leq j \leq n}\left(w_{a_{u_{j}}}\right), \max _{1 \leq j \leq n}\left(u_{a_{j}}\right)\right\rangle \leq\right.$

$$
S_{\mu}\left(\left\langle(\underline{\omega}, \omega, \bar{\omega}) ; w_{\tilde{\omega}}, u_{\tilde{\omega}}\right\rangle\right), i=1,2, \ldots, m ;
$$




$$
\begin{aligned}
& S_{\nu}\left(\left\langle\left(\sum_{j=1}^{n} \underline{a}_{i j} z_{j}, \sum_{j=1}^{n} a_{i j} z_{j}, \sum_{j=1}^{n} \bar{a}_{i j} z_{j}\right) ; \min _{1 \leq j \leq n}\left(w_{\tilde{a}_{j}}\right), \max _{1 \leq j \leq n}\left(u_{\tilde{a}_{j}}\right)\right\rangle \leq\right. \\
& S_{\nu}\left(\left\langle(\underline{\omega}, \omega, \bar{\omega}) ; w_{\tilde{\omega}}, u_{\tilde{\omega}}\right\rangle\right), i=1,2, \ldots, m ; \\
& \sum_{j=1}^{n} z_{j}=1 ; \\
& z_{j} \geq 0, j=1,2, \ldots, n .
\end{aligned}
$$

Step 5: Using Section 1, the problems P21 and P22 can be transformed into problems P23 and P24 respectively.

\section{Problem P23}

$\operatorname{Maximize}\left\{\left(w_{\tilde{v}}\right) \frac{(\underline{v}+2 v+\bar{v})}{4},\left(1-u_{\tilde{v}}\right) \frac{(\underline{v}+2 v+\bar{v})}{4}\right\}$

Subject to

$$
\begin{gathered}
\left(\min _{1 \leq i \leq m}\left(w_{\tilde{a}_{i j}}\right)\right) \frac{\left(\sum_{i=1}^{m} \underline{a}_{i j} y_{i}+2 \sum_{i=1}^{m} a_{i j} y_{i}+\sum_{i=1}^{m} \bar{a}_{i j} y_{i}\right)}{4} \geq \\
\left(w_{\tilde{v}}\right) \frac{(\underline{v}+2 v+\bar{v})}{4}, j=1,2, \ldots, n ; \\
\left(1-\max _{1 \leq i \leq m}\left(u_{\tilde{a}_{j}}\right)\right) \frac{\left(\sum_{i=1}^{m} \underline{a}_{i j} y_{i}+2 \sum_{i=1}^{m} a_{i j} y_{i}+\sum_{i=1}^{m} \bar{a}_{i j} y_{i}\right)}{4} \geq \\
\left(1-u_{\tilde{v}}\right) \frac{(\underline{v}+2 v+\bar{v})}{4}, j=1,2, \ldots, n ;
\end{gathered}
$$

$\underline{v} \leq v$

$v \leq \bar{v}$

$\sum_{i=1}^{m} y_{i}=1$

$y_{i} \geq 0, i=1,2, \ldots, m$.

\section{Problem P24}

$\operatorname{Minimize}\left\{\left(w_{\tilde{\omega}}\right) \frac{(\underline{\omega}+2 \omega+\bar{\omega})}{4},\left(1-u_{\tilde{\omega}}\right) \frac{(\underline{\omega}+2 \omega+\bar{\omega})}{4}\right\}$

Subject to

$$
\begin{gathered}
\left(\min _{1 \leq j \leq n}\left(w_{\tilde{a}_{j}}\right)\right) \frac{\left(\sum_{j=1}^{n} \underline{a}_{i j} z_{j}+2 \sum_{j=1}^{n} a_{i j} z_{j}+\sum_{j=1}^{n} \bar{a}_{i j} z_{j}\right)}{4} \leq \\
\left(1-w_{\tilde{\omega}}\right) \frac{(\underline{\omega}+2 \omega+\bar{\omega})}{4}, i=1,2, \ldots, m ; \\
(1 \leq j \leq n \\
\left(1-u_{\tilde{\omega}}\right) \frac{(\underline{\omega}+2 \omega+\bar{\omega})}{4}, i=1,2, \ldots, m ;
\end{gathered}
$$

$\underline{\omega} \leq \omega$

$\omega \leq \bar{\omega}$

$\sum_{j=1}^{n} z_{j}=1$

$z_{j} \geq 0, j=1,2, \ldots, n$.

Step 6: Assuming $\left(w_{\tilde{v}}\right)(\underline{v}+2 v+\bar{v}) / 4=v_{1}$, $\left(1-u_{\tilde{v}}\right)(\underline{v}+2 v+\bar{v}) / 4=v_{2}$ and $\left(w_{\tilde{\omega}}\right)(\underline{\omega}+2 \omega+\bar{\omega}) / 4=\omega_{1},\left(1-u_{\tilde{\omega}}\right)(\underline{\omega}+2 \omega+\bar{\omega}) / 4=\omega_{2}$ the problems P23 and P24 can be transformed into problems $\mathrm{P} 25$ and $\mathrm{P} 26$ respectively.

\section{Problem P25}

Maximize $\left\{v_{1}, v_{2}\right\}$

Subject to

$\left(\min _{1 \leq i \leq m}\left(w_{\tilde{a}_{j}}\right)\right) \frac{\left(\sum_{i=1}^{m} \underline{a}_{i j} y_{i}+2 \sum_{i=1}^{m} a_{i j} y_{i}+\sum_{i=1}^{m} \bar{a}_{i j} y_{i}\right)}{4} \geq v_{1}, j=1,2, \ldots, n ;$

$\left(1-\max _{1 \leq i \leq m}\left(u_{\tilde{a}_{i j}}\right)\right) \frac{\left(\sum_{i=1}^{m} \underline{a}_{i j} y_{i}+2 \sum_{i=1}^{m} a_{i j} y_{i}+\sum_{i=1}^{m} \bar{a}_{i j} y_{i}\right)}{4} \geq v_{2}, j=1,2, \ldots, n ;$

$v_{2} \geq v_{1}$

$\sum_{i=1}^{m} y_{i}=1$

$y_{i} \geq 0, i=1,2, \ldots, m$.

\section{Problem P26}

Minimize $\left\{\omega_{1}, \omega_{2}\right\}$

Subject to

$\left(\min _{1 \leq j \leq n}\left(w_{\tilde{a}_{j}}\right)\right) \frac{\left(\sum_{j=1}^{n} \underline{a}_{i j} z_{j}+2 \sum_{j=1}^{n} a_{i j} z_{j}+\sum_{j=1}^{n} \bar{a}_{i j} z_{j}\right)}{4} \leq \omega_{1}, i=1,2, \ldots, m ;$

$\left(1-\max _{1 \leq j \leq n}\left(u_{\tilde{a}_{j}}\right)\right) \frac{\left(\sum_{j=1}^{n} \underline{a}_{i j} z_{j}+2 \sum_{j=1}^{n} a_{i j} z_{j}+\sum_{j=1}^{n} \bar{a}_{i j} z_{j}\right)}{4} \leq \omega_{2}, i=1,2, \ldots, m ;$

$\omega_{1} \leq \omega_{2}$;

$\sum_{j=1}^{n} z_{j}=1$

$z_{j} \geq 0, j=1,2, \ldots, n$.

Step 7: Using lexicographic method the optimal solution of the problems P25 and P26 and hence the optimal solution of problems $\mathrm{P} 1$ and $\mathrm{P} 2$ respectively can be obtained as follows. 
Step 7(a): Find the optimal solution $\left\{v_{1}, v_{2}, y_{i}, i=1,2, \ldots, m\right\}$ and $\left\{\omega_{1}, \omega_{2}, z_{j}, j=1,2, \ldots, n\right\}$ of problems P27 and P28. Let it be denoted by $\left\{v_{1}^{0}, v_{2}^{0}, y_{i}^{0}, i=1,2, \ldots, m\right\}$ and $\left\{\omega_{1}^{0}, \omega_{2}^{0}, z_{j}^{0}, j=1,2, \ldots, n\right\}$ respectively.

\section{Problem P27}

Maximize $\left\{v_{1}\right\}$

Subject to

Constraints of problem P25.

\section{Problem P28}

Minimize $\left\{\omega_{1}\right\}$

Subject to

Constraints of problem P26.

Step 7(b): Find the optimal solution $\left\{v_{1}, v_{2}, y_{i}, i=1,2, \ldots, m\right\}$ and $\left\{\omega_{1}, \omega_{2}, z_{j}, j=1,2, \ldots, n\right\}$ of problems $\mathrm{P} 29$ and $\mathrm{P} 30$ respectively. Let it be denoted by $\left\{v_{1}^{*}, v_{2}^{*}, y_{i}^{*}, i=1,2, \ldots, m\right\}$ and $\left\{\omega_{1}^{*}, \omega_{2}^{*}, z_{j}^{*}, j=1,2, \ldots, n\right\}$ respectively.

\section{Problem P29}

Maximize $\left\{v_{2}\right\}$

Subject to

Constraints of problem $\mathrm{P} 25$ with additional constraints $v_{1} \geq v_{1}^{0}$

$v_{2} \geq v_{2}^{0}$.

\section{Problem P30}

Minimize $\left\{\omega_{2}\right\}$

Subject to

Constraints of problem P26 with additional constraints

$\omega_{1} \leq \omega_{1}^{0}$;

$\omega_{2} \leq \omega_{2}^{0}$.

Step 8: Using the maximin strategy $y_{i}^{*}, i=1,2, \ldots m$ and minimax strategy $z_{j}^{*}, j=1,2, \ldots n$, obtained in Step 7(b), the value of intuitionistic fuzzy matrix game for player1 is $E\left(y_{i}^{*}, z_{j}^{*}\right)=\sum_{i=1}^{m} \sum_{j=1}^{n} y_{i}^{*} \tilde{a}_{i j} z_{j}^{*}$.

\section{Exact Optimal Solution of Market Share Problem}

Nan et al. [1, Section 4, pp. 287] solved market share problem to illustrate their proposed method by considering two companies $p_{1}$ and $p_{2}$ and
$\tilde{A}=\left(\begin{array}{ll}\langle(175,180,190) ; 0.6,0.2\rangle & \langle(150,156,158) ; 0.6,0.1\rangle \\ \langle(80,90,100) ; 0.9,0.1\rangle & \langle(175,180,190) ; 0.6,0.2\rangle\end{array}\right)$

as intuitionistic fuzzy payoffs matrix for company $p_{1}$, where $p_{1}$ and $p_{2}$ are regarded as player 1 and player 2 respectively. However, as discussed in Section 3, that Nan et al. [1] have used some mathematical incorrect assumptions. So, the intuitionistic fuzzy optimal solution of market share problem, obtained by Nan et al. [1], is not exact.

In this section, to find the exact optimal solution (maximin strategy $y_{i}^{*}, i=1,2, \ldots m$, minimax strategy $z_{j}^{*}, j=1,2, \ldots n$ for player 1 , player 2 respectively and value of intuitionistic fuzzy matrix game for player 1) of this problem, the corresponding intuitionistic fuzzy linear programming problems $\mathrm{P} 31$ and P32, is solved by the modified method.

\section{Problem P31}

$\operatorname{Maximize}(\tilde{v})$

Subject to

$$
\begin{aligned}
& \langle(175,180,190) ; 0.6,0.2\rangle y_{1}+\langle(80,90,100) ; 0.9,0.1\rangle y_{2} \geq_{I F} \tilde{v} ; \\
& \langle(150,156,158) ; 0.6,0.1\rangle y_{1}+\langle(175,180,190) ; 0.6,0.2\rangle y_{2} \geq_{I F} \tilde{v} ; \\
& y_{1}+y_{2}=1 \\
& y_{1}, y_{2} \geq 0
\end{aligned}
$$

\section{Problem P32}

$\operatorname{Minimize}(\tilde{\omega})$

Subject to

$$
\begin{aligned}
& \langle(175,180,190) ; 0.6,0.2\rangle z_{1}+\langle(150,156,158) ; 0.6,0.1\rangle z_{2} \leq_{I F} \tilde{\omega} ; \\
& \langle(80,90,100) ; 0.9,0.1\rangle z_{1}+\langle(175,180,190) ; 0.6,0.2\rangle z_{2} \leq_{I F} \tilde{\omega} ; \\
& z_{1}+z_{2}=1 ; \\
& z_{1}, z_{2} \geq 0 .
\end{aligned}
$$

Using modified method problems P31 and P32 can be solved as follows:

Step 1: Assuming $\tilde{v}=\langle(\underline{v}, v, \bar{v}) ; 0.6,0.2\rangle$ and $\tilde{\omega}=\langle(\underline{\omega}, \omega, \bar{\omega}) ; 0.6,0.2\rangle$, where

$0.6=\min _{\substack{1 \leq \leq \leq 2 \\ 1 \leq j \leq 2}}\left\{w_{\bar{a}_{j}}\right\}=\{0.6,0.6,0.9,0.6\}$ and

$0.2=\max _{\substack{1<i \leq 2 \\ 1 \leq \leq \leq 2}}\left\{u_{\tilde{a}_{j}}\right\}=\{0.2,0.1,0.1,0.2\}$, the problems P 31 and P32 can be transformed into problems P33 and P34 respectively.

\section{Problem P33}


$\operatorname{Maximize}(\langle(\underline{v}, v, \bar{v}) ; 0.6,0.2\rangle)$

Subject to

$\langle(175,180,190) ; 0.6,0.2\rangle y_{1}+\langle(80,90,100) ; 0.9,0.1\rangle y_{2} \geq_{I F}$

$$
\langle(\underline{v}, v, \bar{v}) ; 0.6,0.2\rangle \text {; }
$$

$\langle(150,156,158) ; 0.6,0.1\rangle y_{1}+\langle(175,180,190) ; 0.6,0.2\rangle y_{2} \geq_{I F}$

$\langle(\underline{v}, v, \bar{v}) ; 0.6,0.2\rangle ;$

$y_{1}+y_{2}=1$

$y_{1}, y_{2} \geq 0$.

\section{Problem P34}

$\operatorname{Minimize}(\langle(\underline{\omega}, \omega, \bar{\omega}) ; 0.6,0.2\rangle)$

Subject to

$\langle(175,180,190) ; 0.6,0.2\rangle z_{1}+\langle(150,156,158) ; 0.6,0.1\rangle z_{2} \leq_{I F}$ $\langle(\underline{\omega}, \omega, \bar{\omega}) ; 0.6,0.2\rangle ;$

$\langle(80,90,100) ; 0.9,0.1\rangle z_{1}+\langle(175,180,190) ; 0.6,0.2\rangle z_{2} \leq_{I F}$

$\langle(\underline{\omega}, \omega, \bar{\omega}) ; 0.6,0.2\rangle ;$

$z_{1}+z_{2}=1$

$z_{1}, z_{2} \geq 0$.

Step 2: Using the arithmetic operation $\left\langle(\underline{a}, a, \bar{a}) ; w_{\tilde{a}}, u_{\tilde{a}}\right\rangle \lambda=\left\langle(\underline{a} \lambda, a \lambda, \bar{a} \lambda) ; w_{\tilde{a}}, u_{\tilde{a}}\right\rangle, \lambda \geq 0 ; \quad$ the problems P33 and P34 can be transformed into problems $\mathrm{P} 35$ and $\mathrm{P} 36$ respectively.

\section{Problem P35}

$\operatorname{Maximize}(\langle(\underline{v}, v, \bar{v}) ; 0.6,0.2\rangle)$

Subject to

$\left(\begin{array}{l}\left\langle\left(175 y_{1}, 180 y_{1}, 190 y_{1}\right) ; 0.6,0.2\right\rangle+ \\ \left\langle\left(80 y_{2}, 90 y_{2}, 100 y_{2}\right) ; 0.9,0.1\right\rangle\end{array}\right) \geq_{I F}\langle(\underline{v}, v, \bar{v}) ; 0.6,0.2\rangle ;$

$\left(\begin{array}{l}\left\langle\left(150 y_{1}, 156 y_{1}, 158 y_{1}\right) ; 0.6,0.1\right\rangle+ \\ \left\langle\left(175 y_{2}, 180 y_{2}, 190 y_{2}\right) ; 0.6,0.2\right\rangle\end{array}\right) \geq_{I F}\langle(\underline{v}, v, \bar{v}) ; 0.6,0.2\rangle$;

$y_{1}+y_{2}=1$;

$y_{1}, y_{2} \geq 0$.

\section{Problem P36}

$\operatorname{Minimize}(\langle(\underline{\omega}, \omega, \bar{\omega}) ; 0.6,0.2\rangle)$

Subject to

$\left(\begin{array}{l}\left\langle\left(175 z_{1}, 180 z_{1}, 190 z_{1}\right) ; 0.6,0.2\right\rangle+ \\ \left\langle\left(150 z_{2}, 156 z_{2}, 158 z_{2}\right) ; 0.6,0.1\right\rangle\end{array}\right) \leq_{I F}\langle(\underline{\omega}, \omega, \bar{\omega}) ; 0.6,0.2\rangle ;$ $\left(\begin{array}{l}\left\langle\left(80 z_{1}, 90 z_{1}, 100 z_{1}\right) ; 0.9,0.1\right\rangle+ \\ \left\langle\left(175 z_{2}, 180 z_{2}, 190 z_{2}\right) ; 0.6,0.2\right\rangle\end{array}\right) \leq_{I F}\langle(\underline{\omega}, \omega, \bar{\omega}) ; 0.6,0.2\rangle ;$

$z_{1}+z_{2}=1$

$z_{1}, z_{2} \geq 0$.

Step 3: Using the arithmetic operation

$\sum_{i=1}^{n}\left\langle\left(\underline{a}_{i}, a_{i}, \bar{a}_{i}\right) ; w_{\tilde{a}_{i}}, u_{\tilde{a}_{i}}\right\rangle=\left\langle\left(\sum_{i=1}^{n} \underline{a}_{i}, \sum_{i=1}^{n} a_{i}, \sum_{i=1}^{n} \bar{a}_{i}\right) ; \min _{1 \leq i \leq n}\left(w_{\tilde{a}_{i}}\right),\right\rangle$

,the problems $\mathrm{P} 35$ and P36 can be transformed into problems P37 and P38 respectively.

\section{Problem P37}

$\operatorname{Maximize}(\langle(\underline{v}, v, \bar{v}) ; 0.6,0.2\rangle)$

Subject to

$$
\begin{aligned}
\begin{array}{r}
\left.\left\langle 175 y_{1}+80 y_{2}, 180 y_{1}+90 y_{2}, 190 y_{1}+100 y_{2}\right) ; 0.6,0.2\right\rangle \geq_{I F} \\
\langle(\underline{v}, v, \bar{v}) ; 0.6,0.2\rangle ;
\end{array} \\
\begin{array}{l}
\left\langle\left(150 y_{1}+175 y_{2}, 156 y_{1}+180 y_{2}, 158 y_{1}+190 y_{2}\right) ; 0.6,0.2\right\rangle \geq_{I F} \\
\quad\langle(\underline{v}, v, \bar{v}) ; 0.6,0.2\rangle ;
\end{array} \\
\begin{array}{l}
y_{1}+y_{2}=1 ; \\
y_{1}, y_{2} \geq 0 .
\end{array}
\end{aligned}
$$

\section{Problem P38}

$\operatorname{Minimize}(\langle(\underline{\omega}, \omega, \bar{\omega}) ; 0.6,0.2\rangle)$

Subject to

$$
\begin{aligned}
& \left\langle\left(175 z_{1}+150 z_{2}, 180 z_{1}+156 z_{2}, 190 z_{1}+158 z_{2}\right) ; 0.6,0.2\right\rangle \leq_{I F} \\
& \langle(\underline{\omega}, \omega, \bar{\omega}) ; 0.6,0.2\rangle ; \\
& \left\langle\left(80 z_{1}+175 z_{2}, 90 z_{1}+180 z_{2}, 100 z_{1}+190 z_{2}\right) ; 0.6,0.2\right\rangle \leq_{I F} \\
& \langle(\underline{\omega}, \omega, \bar{\omega}) ; 0.6,0.2\rangle ; \\
& z_{1}+z_{2}=1 ; \\
& z_{1}, z_{2} \geq 0 .
\end{aligned}
$$

Step 4: Using Section 1, the problems P37 and P38 can be transformed into problems P39 and P40 respectively.

\section{Problem P39}

Maximize

$\left\{S_{\mu}(\langle(\underline{v}, v, \bar{v}) ; 0.6,0.2\rangle), S_{v}(\langle(\underline{v}, v, \bar{v}) ; 0.6,0.2\rangle)\right\}$

Subject to 


$$
\begin{aligned}
& S_{\mu}\left(\left\langle\left(175 y_{1}+80 y_{2}, 180 y_{1}+90 y_{2}, 190 y_{1}+100 y_{2}\right) ; 0.6,0.2\right\rangle\right) \geq \\
& S_{\mu}(\langle(\underline{v}, v, \bar{v}) ; 0.6,0.2\rangle) ; \\
& S_{v}\left(\left\langle\left(175 y_{1}+80 y_{2}, 180 y_{1}+90 y_{2}, 190 y_{1}+100 y_{2}\right) ; 0.6,0.2\right\rangle\right) \geq \\
& S_{v}(\langle(\underline{v}, v, \bar{v}) ; 0.6,0.2\rangle) ; \\
& S_{\mu}\left(\left\langle\left(150 y_{1}+175 y_{2}, 156 y_{1}+180 y_{2}, 158 y_{1}+190 y_{2}\right) ; 0.6,0.2\right\rangle\right) \geq \\
& S_{\mu}(\langle(\underline{v}, v, \bar{v}) ; 0.6,0.2\rangle) ; \\
& S_{v}\left(\left\langle\left(150 y_{1}+175 y_{2}, 156 y_{1}+180 y_{2}, 158 y_{1}+190 y_{2}\right) ; 0.6,0.2\right\rangle\right) \geq \\
& S_{v}(\langle(\underline{v}, v, \bar{v}) ; 0.6,0.2\rangle) ; \\
& y_{1}+y_{2}=1 \text {; } \\
& y_{1}, y_{2} \geq 0 \text {. } \\
& \left\{S_{\mu}(\langle(\underline{\omega}, \omega, \bar{\omega}) ; 0.6,0.2\rangle), S_{v}(\langle(\underline{\omega}, \omega, \bar{\omega}) ; 0.6,0.2\rangle)\right\} \\
& S_{v}(\langle(\underline{\omega}, \omega, \bar{\omega}) ; 0.6,0.2\rangle) ; \\
& S_{\mu}(\langle(\underline{\omega}, \omega, \bar{\omega}) ; 0.6,0.2\rangle) ; \\
& S_{v}(\langle(\underline{\omega}, \omega, \bar{\omega}) ; 0.6,0.2\rangle) ;
\end{aligned}
$$

\section{Problem P40}

Step 5: Using Section 1, the problems P39 and P40 can be transformed into problems P41 and P42 respectively.

\section{Problem P41}

Maximize $\left\{(0.6) \frac{(\underline{v}+2 v+\bar{v})}{4},(1-0.2) \frac{(\underline{v}+2 v+\bar{v})}{4}\right\}$

Subject to

$$
\begin{array}{r}
\text { (0.6) } \frac{\left(175 y_{1}+80 y_{2}+2\left(180 y_{1}+90 y_{2}\right)+190 y_{1}+100 y_{2}\right)}{4} \geq \\
(0.6) \frac{(\underline{v}+2 v+\bar{v})}{4} ;
\end{array}
$$

$$
\begin{aligned}
& \begin{array}{l}
(1-0.2) \frac{\left(175 y_{1}+80 y_{2}+2\left(180 y_{1}+90 y_{2}\right)+190 y_{1}+100 y_{2}\right)}{4} \geq \\
(1-0.2) \frac{(\underline{v}+2 v+\bar{v})}{4} ; \\
(0.6) \frac{\left(150 y_{1}+175 y_{2}+2\left(156 y_{1}+180 y_{2}\right)+158 y_{1}+190 y_{2}\right)}{4} \geq \\
(0.6) \frac{(\underline{v}+2 v+\bar{v})}{4} ; \\
(1-0.2) \frac{\left(150 y_{1}+175 y_{2}+2\left(156 y_{1}+180 y_{2}\right)+158 y_{1}+190 y_{2}\right)}{4} \geq \\
(1-0.2) \frac{(\underline{v}+2 v+\bar{v})}{4} ; \\
\underline{v} \leq v ; \\
v \leq \bar{v} ; \\
y_{1}+y_{2}=1 ; \\
y_{1}, y_{2} \geq 0 .
\end{array}
\end{aligned}
$$

\section{Problem P42}

$\operatorname{Minimize}\left\{(0.6) \frac{(\underline{\omega}+2 \omega+\bar{\omega})}{4},(1-0.2) \frac{(\underline{\omega}+2 \omega+\bar{\omega})}{4}\right\}$

Subject to

$$
\begin{array}{r}
(0.6) \frac{\left(175 z_{1}+150 z_{2}+2\left(180 z_{1}+156 z_{2}\right)+190 z_{1}+158 z_{2}\right)}{4} \leq \\
(0.6) \frac{(\underline{\omega}+2 \omega+\bar{\omega})}{4} ; \\
(1-0.2) \frac{\left(175 z_{1}+150 z_{2}+2\left(180 z_{1}+156 z_{2}\right)+190 z_{1}+158 z_{2}\right)}{4} \leq \\
(1-0.2) \frac{(\underline{\omega}+2 \omega+\bar{\omega})}{4} ;
\end{array}
$$

(0.6) $\frac{\left(80 z_{1}+175 z_{2}+2\left(90 z_{1}+180 z_{2}\right)+100 z_{1}+190 z_{2}\right)}{4} \leq$

$$
\text { (0.6) } \frac{(\underline{\omega}+2 \omega+\bar{\omega})}{4} \text {; }
$$

$$
\begin{array}{r}
(1-0.2) \frac{\left(80 z_{1}+175 z_{2}+2\left(90 z_{1}+180 z_{2}\right)+100 z_{1}+190 z_{2}\right)}{4} \leq \\
(1-0.2) \frac{(\underline{\omega}+2 \omega+\bar{\omega})}{4} ;
\end{array}
$$

$\underline{\omega} \leq \omega$

$\omega \leq \bar{\omega}$;

$z_{1}+z_{2}=1$

$z_{1}, z_{2} \geq 0$.

Step 6: Assuming $(0.6)(\underline{v}+2 v+\bar{v}) / 4=v_{1}$,

$(1-0.2)(\underline{v}+2 v+\bar{v}) / 4=v_{2}$ and

$(0.6)(\underline{\omega}+2 \omega+\bar{\omega}) / 4=\omega_{1}$, 
$(1-0.2)(\underline{\omega}+2 \omega+\bar{\omega}) / 4=\omega_{2}$, the problems P41 and P42 can be transformed into problems P43 and P44 respectively.

\section{Problem P43}

Maximize $\left\{v_{1}, v_{2}\right\}$

Subject to

(0.6) $\frac{\left(175 y_{1}+80 y_{2}+2\left(180 y_{1}+90 y_{2}\right)+190 y_{1}+100 y_{2}\right)}{4} \geq v_{1}$;

$(1-0.2) \frac{\left(175 y_{1}+80 y_{2}+2\left(180 y_{1}+90 y_{2}\right)+190 y_{1}+100 y_{2}\right)}{4} \geq v_{2}$;

(0.6) $\frac{\left(150 y_{1}+175 y_{2}+2\left(156 y_{1}+180 y_{2}\right)+158 y_{1}+190 y_{2}\right)}{4} \geq v_{1}$;

$(1-0.2) \frac{\left(150 y_{1}+175 y_{2}+2\left(156 y_{1}+180 y_{2}\right)+158 y_{1}+190 y_{2}\right)}{4}$

$\geq v_{2}$

$v_{2} \geq v_{1}$

$y_{1}+y_{2}=1$

$y_{1}, y_{2} \geq 0$.

\section{Problem P44}

Minimize $\left\{\omega_{1}, \omega_{2}\right\}$

Subject to

$(0.6) \frac{\left(175 z_{1}+150 z_{2}+2\left(180 z_{1}+156 z_{2}\right)+190 z_{1}+158 z_{2}\right)}{4} \leq \omega_{1} ;$
$(1-0.2) \frac{\left(175 z_{1}+150 z_{2}+2\left(180 z_{1}+156 z_{2}\right)+190 z_{1}+158 z_{2}\right)}{4}$

$\begin{aligned} & \leq \omega_{2} \\ &(0.6) \frac{\left(80 z_{1}+175 z_{2}+2\left(90 z_{1}+180 z_{2}\right)+100 z_{1}+190 z_{2}\right)}{4} \leq \omega_{1}\end{aligned}$

$(1-0.2) \frac{\left(80 z_{1}+175 z_{2}+2\left(90 z_{1}+180 z_{2}\right)+100 z_{1}+190 z_{2}\right)}{4}$ $\leq \omega_{2}$

$\omega_{1} \leq \omega_{2}$

$z_{1}+z_{2}=1$

$z_{1}, z_{2} \geq 0$.

Step 7: Using lexicographic method the optimal solution of problems P43 and P44 and hence the optimal solution of problems P31 and P32 respectively can be obtained as follows.
Step 7(a): The optimal solution of problems P45 and $\mathrm{P} 46$ is $\left\{v_{1}=\frac{36291}{376}, v_{2}=\frac{36291}{376}, y_{1}=\frac{73}{94}, y_{2}=\frac{21}{94}\right\}$ and $\left\{\omega_{1}=\frac{36291}{376}, \omega_{2}=\frac{12097}{94}, z_{1}=\frac{21}{94}, z_{2}=\frac{73}{94}\right\}$

respectively.

\section{Problem P45}

Maximize $\left\{v_{1}\right\}$

Subject to

Constraints of problem P43.

\section{Problem P46}

Minimize $\left\{\omega_{1}\right\}$

Subject to

Constraints of problem P44.

Step 7 (b): The exact optimal solution of problems P47 and P48 and hence of problems P31 and P32 is $\left\{v_{1}=\frac{36291}{376}, v_{2}=\frac{12097}{94}, y_{1}=\frac{73}{94}, y_{2}=\frac{21}{94}\right\}$ and $\left\{\omega_{1}=\frac{36291}{376}, \omega_{2}=\frac{12097}{94}, z_{1}=\frac{21}{94}, z_{2}=\frac{73}{94}\right\}$ respectively.

\section{Problem P47}

Maximize $\left\{v_{2}\right\}$

Subject to

Constraints of problem P43 with additional constraints $v_{1} \geq \frac{36291}{376}$;

$v_{2} \geq \frac{36291}{376}$.

\section{Problem P48}

Maximize $\left\{\omega_{2}\right\}$

Subject to

Constraints of problem P44 with additional constraints $\omega_{1} \leq \frac{36291}{376}$

$\omega_{2} \leq \frac{12097}{94}$.

Step 8: Using the maximin strategy $y_{1}^{*}=\frac{73}{94}, y_{2}^{*}=\frac{21}{94}$ and minimax strategy $z_{1}^{*}=\frac{21}{94}, z_{2}^{*}=\frac{73}{94}$, 
obtained in Step 7(b), the exact solution of intuitionistic fuzzy matrix game for the market share problem of company $p_{1}$ is

$E\left(y_{i}^{*}, z_{j}^{*}\right)=\left\langle\left(\frac{342795}{2209}, \frac{711447}{4418}, \frac{734311}{4418}\right) ; 0.6,0.2\right\rangle$

$=\langle(155.18,161.03,166.21) ; 0.6,0.2\rangle$ as shown in the following Fig. 1.

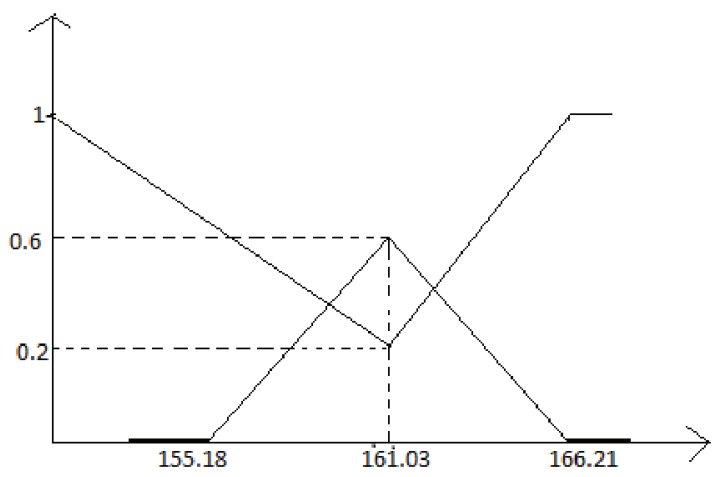

Fig.1: The exact solution of intuitionistic fuzzy matrix game for the market share problem of company $p_{1}$

\section{Conclusion}

The error in the existing method [1] is pointed out and the modified method is proposed to find the exact solution of such matrix games in which payoffs are represented by triangular intuitionistic fuzzy numbers.

\section{Acknowledgements}

The authors would like to thank Editor-in-Chief and anonymous referees for various suggestions which have led to an improvement in both the quality and clarity of the paper. The authors also would like to acknowledge the adolescent inner blessings of Mehar (lovely daughter of cousin sister of Dr. Amit Kumar). They believe that Mata Vaishno Devi has appeared on the earth in the form of Mehar and without her blessings it was not possible to think the ideas presented in this paper. The first author acknowledge the financial support given to her by Department of Science and Technology under INSPIRE program for research students [IF130759] to complete doctoral program and would like to thank God for not letting her down at the time of crisis and showing her the silver lining in the dark clouds.

\section{References}

1. J.-X. Nan, D.- F. Li and M.- J. Zhang, A lexicographic method for matrix games with payoffs of triangular intuitionistic fuzzy numbers, International Journal of Computational Intelligence Systems 3(3) (2010) 280289. 